\title{
Thermal Conductivity Modeling of U-Mo/Al Dispersion Fuel
}

Yeon Soo Kim ${ }^{1}$, Byoung Jin Cho ${ }^{2}$, Dong-Seong Sohn ${ }^{2 *}$, Jong Man Park ${ }^{3}$

1: Argonne National Laboratory, 9700 South Cass Avenue, Argonne, IL 60439 USA

2: Ulsan National Institute of Science and Technology, 50 UNIST-gil, Eonyang-eup, Ulju-gun, Ulsan, Republic of Korea

3: Korea Atomic Energy Research Institute, 989-111 Daedeok-daero, Yuseong-gu, Daejon 305-353, Repubic of Korea

* Corresponding author: email

dssohn@unist.ac.kr 


\begin{abstract}
A dataset for the thermal conductivity of U-Mo/Al dispersion fuel made available by KAERI was reanalyzed. Using this dataset, an analytical model was obtained by expanding the Bruggeman model. The newly developed model incorporates thermal resistances at the interface between the U-Mo particles and the Al matrix and the defects within the Al matrix (grain boundaries, cracks, and dislocations).The interfacial resistances are expressed as functions of U-Mo particle size and Al grain size obtained empirically by fitting to measured data from KAERI. The model was then validated against an independently measured dataset from ANL.
\end{abstract}

Key words: U-Mo fuel, U-Mo/Al dispersion fuel, meat thermal conductivity, composite material thermal conductivity, modeling 
1. Introduction

Due to its high density and good irradiation stability, U-Mo particles dispersed in an Al matrix (U-Mo/Al) is a promising fuel for conversion of the research reactors that currently use HEU fuels to LEU fuels. The current fuels such as U-oxides, U-Al intermetallics, and USi intermetallics dispersed in an Al matrix have a lower U-density than U-Mo/Al. The typical size of the U-Mo fuel kernels is about $70 \mu \mathrm{m}$. The most common method to produce U-Mo powders is the atomization method, in which a U-Mo melt is poured onto a spinning disc that sprays U-Mo droplets around [1]. Because the droplets harden while suspended in the air, the particle shape is spherical. An older, and less popular, method is to grind U-Mo chips to obtain smaller particles [2]. Unlike the atomization method, this method produces particles with an irregular shape. The fueled zone (the mixture zone of U-Mo particles and Al matrix) is termed the 'fuel meat' (see Fig. 1). The volume fraction of U-Mo fuel in the meat needed is determined by the required fissile content in the fuel plate or rod. This has a typical range of 10 to $55 \%$ in most applications. The upper limit is set by the current technological limit of the hot rolling method used for fuel plate fabrication [4].

The primary metric for fuel performance is the temperature of the fuel, which is determined by the thermal conductivity. Therefore, any predictive model for fuel performance requires accurate values for the thermal conductivity. The frequently adopted model for this purpose is the Bruggeman model [5] or a derivation of this model [6]. These models, in turn, originate from the Maxwell equation [7]. 
The Bruggeman model assumes perfect contact between the dispersed particles and the matrix in the composite medium. It also assumes the matrix is a defect-free monolithic continuum. In reality, the U-Mo particle surface has a thin oxide layer, so the contact between the $\mathrm{U}-\mathrm{Mo}$ and $\mathrm{Al}$ is only partial in a $\mathrm{U}-\mathrm{Mo} / \mathrm{Al}$ dispersion fuel. Additionally, the $\mathrm{Al}$ matrix is a thermally imperfect medium because it is made of $\mathrm{Al}$ powder, which results in grain boundaries. Therefore, the Bruggeman model gives a substantially higher prediction for thermal conductivity than the measured data. In addition, the theoretical model lacks the capability to include the effect of the fuel particle size, or the surface area to volume ratio, to take into account the thermal resistance at the fuel-to-matrix interface.

Measurement data for thermal conductivity of $\mathrm{U}-\mathrm{Mo} / \mathrm{Al}$ is scarce in the literature. Only two sets of data are available in the open literature. One was reported by Korea Atomic Energy Research Institute (KAERI), Korea [8], for which one of the authors of the present study (JMP) co-worked. The other was commissioned by Argonne National Laboratory (ANL) [9]. The latter has not been published in the open literature. In this paper, we refer to the former as KAERI data and the latter as ANL data. The former was measured for atomized spherical U-Mo powder dispersion, whereas the latter was obtained for irregular shaped ground powder dispersion. Due to the irregular U-Mo particle shape, the ANL data may have higher porosity in the meat, potentially resulting in a lower thermal conductivity in the meat. For both datasets, thermal diffusivity and heat capacity were measured, and then thermal conductivity was derived from the measured data.

In this paper, we have corrected the KAERI data to include temperature-dependent density, as the original data was obtained using room temperature density. Another adjustment made 
was to modify the data to eliminate the effects of inhomogeneous U-Mo particle distribution in the samples. The Bruggeman model was expanded to better fit the measured data from KAERI. The KAERI data was adopted for the analyses because it was systematically documented. The ANL data was used to validate the newly developed model.

\section{Theoretical models}

One of the earliest thermal conductivity models for a composite medium is the Maxwell model, derived for a cube containing a spherical particle [7]. The Maxwell model has the following assumptions: the dispersed particles are spherical, the particles do not thermally interact, and the interfacial resistance between the particles and the matrix is negligible.

Because of these assumptions, this model applies better to cases with a low volume fraction of the dispersant. The effective thermal conductivity of the composite by the Maxwell model is expressed as follows:

$\mathrm{k}_{\mathrm{e}}=\mathrm{k}_{\mathrm{m}}\left[\frac{2 \mathrm{k}_{\mathrm{m}}+\mathrm{k}_{\mathrm{p}}-2 \mathrm{~V}_{\mathrm{p}}\left(\mathrm{k}_{\mathrm{m}}-\mathrm{k}_{\mathrm{p}}\right)}{2 \mathrm{k}_{\mathrm{m}}+\mathrm{k}_{\mathrm{p}}+\mathrm{V}_{\mathrm{p}}\left(\mathrm{k}_{\mathrm{m}}-\mathrm{k}_{\mathrm{p}}\right)}\right]$

where the subscripts $m$ and $p$ denote the matrix and the particle, respectively.

To overcome the limitations of the Maxwell model, Bruggeman devised an alternative means by extending the Maxwell model. The Bruggeman model considers an infinite number of 
small additions to a homogeneous mixture of the dispersant and matrix. The Bruggeman model takes the following form:

$\mathrm{k}_{\mathrm{e}}=\frac{1}{4}\left[\mathrm{~A}+\left(\mathrm{A}^{2}+8 \mathrm{k}_{\mathrm{p}} \mathrm{k}_{\mathrm{m}}\right)^{\frac{1}{2}}\right]$

where $A=\left(3 v_{p}-1\right) k_{p}+\left(3 v_{m}-1\right) k_{m}$. Here $v$ is the volume fraction, and $m$ and $p$ have the same meaning as in Eq. (1).

The prediction accuracy of the Bruggeman model improves when the extent of the reaction between the dispersant and the matrix at the interfaces decreases [9]. When a reaction occurs, the Bruggeman model in general overpredicts. The Bruggeman model also assumes perfect bonding between the dispersant and matrix phases, which is not the case in the U-Mo/Al dispersion fuels studied here.

3. Meat constituent thermal conductivities

3.1 U-Mo alloy thermal conductivity

Kim proposed an empirical correlation for thermal conductivity of U-Mo alloys with respect to Mo content in a data compilation report [10]. This correlation was examined more thoroughly for U-Zr alloys in [11], and modified to follow the form in Eq. (3):

$$
\mathrm{k}_{\mathrm{U}-\mathrm{Mo}}=\left(1-\sqrt{1-\mathrm{x}_{\mathrm{Mo}}}\right) \mathrm{k}_{\mathrm{Mo}}+\sqrt{1-\mathrm{x}_{\mathrm{Mo}}}\left\{\left(1-\mathrm{x}_{\mathrm{Mo}}\right) \mathrm{k}_{\mathrm{U}}+\mathrm{x}_{\mathrm{Mo}} \mathrm{k}_{\mathrm{c}, \mathrm{Mo}}\right\}
$$


where $\mathrm{k}_{\mathrm{U}-\mathrm{Mo}}$ is $\mathrm{U}-\mathrm{Mo}$ thermal conductivity in $\mathrm{W} / \mathrm{m}-\mathrm{K}, \mathrm{x}_{\mathrm{Mo}}$ is the Mo content in weight fraction, and $\mathrm{k}_{\mathrm{c}, \mathrm{Mo}}$ is given by

$$
\begin{aligned}
\mathrm{k}_{\mathrm{c}, \mathrm{Mo}}= & -274.4+985.2 \mathrm{x}_{\mathrm{Mo}}-1.941 \times 10^{3} \mathrm{x}_{\mathrm{Mo}}{ }^{2}+3.640 \times 10^{-2} \mathrm{~T}+ \\
& 7.365 \times 10^{-5} \mathrm{~T}^{2}+5.793 \times 10^{-2} \mathrm{x}_{\mathrm{Mo}} \mathrm{T}
\end{aligned}
$$

where $\mathrm{T}$ is in $\mathrm{K}$.

\subsection{Al matrix thermal conductivity}

Aluminum 1050 was used for the matrix of the U-Mo/Al dispersion samples. This aluminum alloy is considered industrially pure because it is $99.75 \% \mathrm{Al}$ with only $0.25 \%$ total impurity.

It is known that the thermal conductivity of pure aluminum increases with temperature from 25 to $127^{\circ} \mathrm{C}$, and then decreases until the melting point at $660{ }^{\circ} \mathrm{C}$ [12]. Using Touloukian's compilation of the thermal conductivity of $\mathrm{Al}$ [12], we developed an equation for the $\mathrm{Al}$ thermal conductivity as a function of temperature.

$\mathrm{k}_{\mathrm{Al}}=144.3+0.6518 \mathrm{~T}-1.531 \times 10^{-3} \mathrm{~T}^{2}+1.458 \times 10^{-6} \mathrm{~T}^{3}-5.188 \times 10^{-10} \mathrm{~T}^{4}$

4. $\quad$ Measured data

4.1 KAERI Data 
The KAERI thermal conductivity data was obtained using samples cut out of the meat region of the fuel rod cross sections. The $2.5 \mathrm{~mm}$ x $2.5 \mathrm{~mm}$ samples were cut from fuel rods that were fabricated by co-extrusion of the fuel meat and $\mathrm{Al}$ cladding. The U-Mo/Al dispersion fuel meat was made with KAERI-fabricated atomized U-Mo particles. The average particle size was about $65 \mu \mathrm{m}$, with $70 \%$ of fuel particles in the $45-150 \mu \mathrm{m}$ range and $30 \%$ fines smaller than $45 \mu \mathrm{m}$. Although not measured for the present work, porosity was considered negligible because the fuel rods were fabricated by the co-extrusion method. From experience, this method produces rods with negligible porosity [13],[14]. However, the fuel particle distribution in the meat is not completely uniform. The original paper reporting the KAERI data [8] did not show a sample cross-section image, so it is impossible to assess the homogeneity of the particle distribution in the thermal diffusivity samples. The uncertainties in the measured thermal conductivity data are believed to be caused chiefly by the uncertainty in the U-Mo volume fraction. However, the original dataset was lack of information that can be used to estimate uncertainties. In the present study, therefore, no attempt was made to assess the uncertainty in the measured data.

The KAERI data includes three alloy types with different Mo contents: U-6Mo, U-8Mo and U-10Mo; and four fuel meat types with different fuel volume fractions: 10, 30, 40 and 50\%.

Using the laser flash method, thermal diffusivity was measured from 25 to $500{ }^{\circ} \mathrm{C}$. Using differential scanning calorimetry (DSC), the heat capacity was measured from 25 to $380{ }^{\circ} \mathrm{C}$. Thermal conductivity was calculated using Eq. (6) for the temperature range $25-380{ }^{\circ} \mathrm{C}$.

$\mathrm{k}=\rho \alpha \mathrm{c}_{\mathrm{p}}$ 
where $\rho$ is the density, $\alpha$ is the thermal diffusivity and $\mathrm{c}_{\mathrm{P}}$ is specific heat capacity. The authors of the KAERI data neglected the change in density as a function of temperature when applying Eq. (6), using only the room temperature density value. The KAERI data is given in Table 1.

4.2 ANL data

ANL commissioned TPRL, Inc. to measure the thermal conductivity of U-Mo/Al dispersion meat. The samples were made at ANL by using a ground U-10Mo powder. Two meat types with different fuel volume fractions were studied. From the meat bulk densities, we estimated the fuel volume fractions of the samples as 39 and 50\%. Because of the use of ground powder, it is reasonable to assume that the samples contain some porosity, particularly in the higher fuel volume fraction case.

The thermal conductivity was calculated from density, thermal diffusivity and specific heat capacity using Eq. (6), similarly for the KAERI data. Unlike the KAERI data, the temperature-dependent density was used for the ANL data. However, information on the homogeneity of the U-Mo distribution was not given. The ANL data is also provided in

\section{Table 1.}

5. KAERI data correction

5.1 Temperature-dependent density correction 
5.1.1 Al thermal expansion and temperature-dependent density

The recommended values for the linear thermal expansion of Al were made available by Touloukian and co-workers [15] and they are given in Table 2. Using this data, we developed a correlation for linear thermal expansion as a function of temperature as follows:

$\Delta \mathrm{L} / \mathrm{L}_{0}=-0.676+2.32 \times 10^{-3} \mathrm{~T}-2.80 \times 10^{-7} \mathrm{~T}^{2}+7.91 \times 10^{-10} \mathrm{~T}^{3}$

where $\mathrm{T}$ is temperature in $\mathrm{K}$ in the range of $293 \leq \mathrm{T} \leq 900 \mathrm{~K}$.

$\mathrm{Al}$ density at temperatures other than room temperature can be calculated using the linear thermal expansion given in Eq. (7) as follows:

$\rho=\left(1+\Delta \mathrm{L} / \mathrm{L}_{0}\right)^{-3} \rho_{0}$

where $\rho_{0}$ is the density at room temperature $2.7 \mathrm{~g} / \mathrm{cm}^{3}$.

5.1.2 U-Mo thermal expansion and temperature-dependent density 
Thermal expansion data for $\gamma$-phase U-Mo alloys can be found in several sources (see [10] and references therein). The mean linear thermal expansion coefficient of U-Mo monotonically increases as a function of temperature as follows:

$\bar{\alpha}_{1}=7.91+1.21 \times 10^{-2} \mathrm{~T}$

where $T$ is the temperature in $\mathrm{K}$ in the range $293-773 \mathrm{~K}$ and $\bar{\alpha}_{1}$ is in $10^{-6} / \mathrm{K}$.

U-Mo density at temperatures other than room temperature can be calculated by

$\rho=\left[1+\frac{1-\left(1+\Delta \mathrm{T} \bar{\alpha}_{1}\right)^{3}}{\left(1+\Delta \mathrm{T} \bar{\alpha}_{1}\right)^{3}}\right] \rho_{0}$

where $\rho_{0}$ is the density at $\mathrm{T}_{0}=298 \mathrm{~K}$, and $\Delta \mathrm{T}=\mathrm{T}-\mathrm{T}_{0}$.

\subsubsection{U-Mo/Al dispersion temperature-dependent density}

The density of U-Mo/Al dispersion fuel was calculated by the following mixture equation:

$\rho_{\mathrm{U}-\mathrm{Mo} / \mathrm{Al}}=\mathrm{V}_{\mathrm{U}-\mathrm{Mo}} \rho_{\mathrm{U}-\mathrm{Mo}}+\left(1-\mathrm{v}_{\mathrm{U}-\mathrm{Mo}}\right) \rho_{\mathrm{Al}}$

where $\mathrm{v}$ is the volume fraction. The temperature-dependent densities are used for Eq. (11). 


\subsubsection{Comparison of the modified KAERI data with Bruggeman model predictions}

Using Eqs. (6) and (8), the KAERI thermal conductivity data for U-Mo/Al dispersion samples were modified to include temperature-dependent density. The Bruggeman model given in Eq. (2) together with the U-Mo alloy thermal conductivity and Al thermal conductivity given in Eq. (3) and Eq. (5), respectively, were employed to predict the thermal conductivity of U-Mo/Al dispersion samples. The results were compared to the modified measured KAERI data (Fig. 2 - Fig. 4). The measured and predicted results are inconsistent with each other. Moreover, it appears that there is no systematic trend in this inconsistency, indicating that modifying the data to include temperature-dependent density did not correct the measured data. The 10 vol.\% case exhibits the largest discrepancy between the measured and predicted data. A possible reason for this disagreement may be due to the inhomogeneous distribution of U-Mo particles in the meat. The 10 vol.\% case is expected to have the highest level of inhomogeneity due to clustering of the U-Mo particles [16].

5.2 Correction for inhomogeneity in U-Mo distribution in the KAERI samples

The effect of the Mo content on the thermal conductivity of U-Mo/Al dispersion is small in the $6-10 \mathrm{wt} \%$ Mo range because the $\mathrm{Al}$ matrix is the predominant determinant of meat thermal conductivity. Instead, the uniformity of the U-Mo particle distribution in the meat has a greater effect. As is discussed in subsection 4.1, the typical dispersion meat is not uniform in distribution. Due to the small size of the laser flash samples, the U-Mo particle distribution 
is expected to have low uniformity. The difference between the real U-Mo volume fraction in the samples and the nominal value reported in the original publication is not known.

Additionally, cross-sectional images of the samples that could show the particle distribution are unavailable. To overcome this potential source of error, the thermal diffusivities measured in samples with the same U-Mo volume fraction, but different Mo content were averaged. This value was then used for all samples with the same U-Mo volume fraction. An example of this method is shown in Fig. 5. This method reduces the effect of local inhomogeneities, and is justified because the effect of Mo content on the meat thermal conductivity is small.

Fig. 6 compares the results obtained using the averaging method and Bruggeman model predictions for U-8Mo/Al. From Eq. (3), one can predict that the thermal conductivity of UMo decreases with the Mo content. Therefore, for the same fuel volume fraction in the meat, the averaged thermal conductivity between $\mathrm{U}-6 \mathrm{Mo} / \mathrm{Al}, \mathrm{U}-8 \mathrm{Mo} / \mathrm{Al}$ and $\mathrm{U}-10 \mathrm{Mo} / \mathrm{Al}$ is expected to be close to that of $\mathrm{U}-8 \mathrm{Mo} / \mathrm{Al}$.

It is noticeable in Fig. 6 that the measured data is systematically lower than the predicted values.

6. New model development

\subsection{Expanded Bruggeman model}

The Bruggeman model, as other theoretical models commonly do, assumes that the interface between the U-Mo particle and Al matrix is in perfect contact and the matrix is a defect-free 
monolithic continuum. However, it is known that the U-Mo particle surface is covered with a $\sim 1 \mu \mathrm{m}$ thick oxide film. Hence, the interface is microscopically only partially bonded.

Additionally, the Al matrix includes defects such as grain boundaries, cracks, and dislocations [16] because it is sintered during co-extrusion from an Al powder shown in Fig. 7.

The Bruggeman model was modified to incorporate thermal resistance factors to overcome limitations stemming from the assumptions applicable only in ideal conditions. The effective thermal conductivity of $\mathrm{U}-\mathrm{Mo} / \mathrm{Al}$ dispersion meat is expressed by

$$
\begin{aligned}
& \mathrm{k}_{\mathrm{e}}=\frac{1}{4}\left[\mathrm{~A}+\left(\mathrm{A}^{2}+8 \mathrm{k}_{\mathrm{p}}^{*} \mathrm{k}_{\mathrm{m}}^{*}\right)^{\frac{1}{2}}\right] \\
& \mathrm{A}=\left(3 \mathrm{v}_{\mathrm{p}}-1\right) \mathrm{k}_{\mathrm{p}}^{*}+\left(3 \mathrm{v}_{\mathrm{m}}-1\right) \mathrm{k}_{\mathrm{m}}^{*}
\end{aligned}
$$

where $\mathrm{p}$ and $\mathrm{m}$ denotes particle and matrix, respectively, and $\mathrm{v}$ is the volume fraction. The interfacial thermal resistance is reflected in

$$
\mathrm{k}_{\mathrm{i}}^{*}=\left(1-\alpha_{\mathrm{i}}\right) \mathrm{k}_{\mathrm{i}}
$$

where $\mathrm{i}=\mathrm{p}$ or $\mathrm{m}$, which are defined the same as in Eq. (1). $\mathrm{k}_{\mathrm{p}}^{*}$ is the reduced thermal conductivity of U-Mo due to interface resistance between the U-Mo particle and the Al matrix. $\mathrm{k}_{\mathrm{m}}^{*}$ is the reduced thermal conductivity of $\mathrm{Al}$ matrix due to defects such as grain boundaries, cracks, and dislocations in the Al matrix. $\alpha_{i}$ is the thermal conductivity reduction factor, defined as follows [17]-[20]: 
$\alpha_{i}=\frac{k_{i}}{r_{i}} R_{i}$

where $\mathrm{k}$ is the thermal conductivity of $\mathrm{U}-\mathrm{Mo}$ or the Al matrix, and $\mathrm{r}$ is the U-Mo particle size or the grain size of the Al matrix. The interfacial thermal resistance $\left(\mathrm{R}_{\mathrm{i}}\right)$ is defined by:

$$
R_{i}=a_{i}\left[1-\exp \left(-\frac{b_{i}}{T}\right)\right]
$$

where $\mathrm{T}$ is the temperature, and $\mathrm{a}$ and $\mathrm{b}$ are constants. These constants are determined by fitting to the KAERI data, as will be discussed in section 6.2. It is worth noting that the current model can predict the effect of the U-Mo particle size.

6.2 Model constant determination

The constants were obtained by fitting the new model to the KAERI data modified by the averaging method. The average U-Mo particle size of $65 \mu \mathrm{m}$ and an Al grain size of $15 \mu \mathrm{m}$ were used. Several iterations were made. The best fit was found when the values given in Table 3 were used.

The predictions using this modified model are compared with the corrected KAERI data in

Fig. 8. There is a noticeable improvement in the consistency between predicted and measured 
values. Considering that the measured data still contain uncertainties, the level of consistency in the prediction suggests that the new model is reliable.

\section{Model validation}

The new model was applied to the 50 vol.\% case from the ANL data [9]. This case had a more typical variation of thermal conductivity with temperature than the 39 vol. $\%$ case. In Fig. 9, a comparison is shown between the model prediction and the measured data. It is noticeable that the temperature dependence of the thermal conductivity is weaker for the predicted values than the measured values. As proposed by the author of the ANL data [9], this is likely due to the healing of the cracks in the ground U-Mo powder. During powder fabrication, the ground powder is subject to substantial mechanical stresses, producing many more defects than in the atomized case. These defects reduce the overall thermal conductivity. However, as the temperature increases more and more of these defects are healed, resulting in an increasing thermal conductivity..

The ANL data appears to be generally over-estimated, especially considering the porosity in the meat. It is known that fuel meat fabricated with ground powders has greater porosity than that produced with an atomized powder [2]. The effect of porosity on the thermal conductivity of the meat can be estimated using the models available in the literature [21],[22]. If $5 \%$ porosity is assumed, a $10 \%$ reduction in thermal conductivity is calculated.

Considering the known measurement uncertainties in the ANL data, the prediction made by the new model matches remarkably well. Therefore, until more measured data become available and a necessity arises for a revision, the use of this new model is recommended. 


\section{Conclusions}

Two datasets for thermal conductivity of U-Mo/Al dispersion are available. One was published by KAERI, South Korea and the other was by ANL. The KAERI data was reexamined and corrected. The Bruggeman model was expanded to better fit the corrected KAERI data. Improved from the Bruggeman model, the current model additionally takes into account the contact resistance between the U-Mo particles and the $\mathrm{Al}$ in the meat, and the defects such as grain boundaries and cracks between the Al grains in the Al matrix. The current model predict the effect of the U-Mo particle size. The current model was then validated against the ANL data. The prediction by this new model was in reasonable accord with the ANL data, indicating that the model is reliable. 


\section{Acknowledgments}

The authors thank Dr. Ho Jin Ryu of KAIST, South Korea, for providing the image used in Fig. 1 that was taken when he worked at KAERI. Dr. S.H. Lee of KRISS is also acknowledged for the measurement data. This work was supported by the National Research Foundation of Korea (NRF) grant funded by the Korean government (Ministry of Education, Science and Technology) under contract number 2011- 0031771 and in part by the U.S.

Department of Energy, National Nuclear Safety Administration (NNSA), Office of Material Management and Minimization (NA-23) Reactor Conversion Program under Contract No. DE-AC-02-06CH11357 between UChicago Argonne, LLC and the US Department of Energy. 


\section{References}

[1] C.K. Kim, J.M. Park, H.J. Ryu, Nucl. Eng. Technol. 39, 617, 2007.

[2] Yeon Soo Kim, Uranium Intermetallic Fuels (U-Al, U-Si, U-Mo). In: R.J.M, Konings (ed.), Comprehensive Nuclear Materials, volume 3, pp. 391-422, Amsterdam, Elsevier (2012).

[3] H.J. Ryu, Y.S. Han, J.M Park, S.D. Park, C.K. Kim, "Reaction layer growth and reaction heat of $\mathrm{U}-\mathrm{Mo} / \mathrm{Al}$ dispersion fuels using centrifugally atomized powders," J. Nucl. Mater. 321 (2003) 210.

[4] T. Wiencek, ANL/RERTR/TM-11, Argonne National Laboratory, 1995.

[5] D.A.G. Bruggeman, "Dielectric constant and conductivity of mixtures of isotopic materials," Ann. Phys., pp. 636-664, 1935.

[6] Z. Hashin, S. Shtrikman, J. Appl. Phys. 33 (1962) 2135.

[7] J.C. Maxwell, A treatise on Electricity and Magnetism, Dover Publications, Inc., 1904.

[8] S.H. Lee, J.M. Park, C.K. Kim, “Thermophysical Properties of U-Mo/Al Alloy Dispersion Fuel Meats,” Intern. J. Thermophys. 28 (2007) 1578.

[9] R.E. Taylor, Thermophysical properties of U-Mo core: A report to Argonne National Laboratory, TPRL, Inc., West Lafayette, IN, 2000.

[10] J. Rest, Yeon Soo Kim, G.L. Hofman, U-Mo fuels handbook, ANL-09/31, Argonne National Laboratory, 2009. 
[11] Yeon Soo Kim, T.W. Cho, D.-S. Sohn, “Thermal conductivities of actinides (U, Pu, $\mathrm{Np}, \mathrm{Cm}, \mathrm{Am}$ ) and uranium alloys (U-Zr, U-Pu-Zr and U-Pu-TRU-Zr),” J. Nucl. Mater. 445 (2014) 272.

[12] Y. S. Touloukian, R.W. Powell, C.Y. Ho, P.G. Klemens, Thermophysical properties of matter, Thermal conductivity, Vol. 1,TPRC, IFI/Plenum, New York, 1970.

[13] J.M. Park, Personal communication, 2011.

[14] H.J. Ryu, J.M. Park, Y.J. Jeong, K.H. Lee, Y.S. Lee, C.K. Kim, Yeon Soo Kim, "Post-irradiation analyses of U-Mo dispersion fuel rods of KOMO tests at HANARO," Nucl. Eng. Technol., 45 (2013) 847-858.

[15] Y.S. Touloukian, R.K. Kirby, R.E. Taylor, T.Y.R. Lee, “Thermophysical properties of matter,’Thermal expansion, vol.13, TPRC, IFI/Plenum, New York, 1977.

[16] J.M. Park, Personal communication, 2014.

[17] T.W. Cho, D-S. Sohn, Yeon Soo Kim, J. Nucl. Sci. Technol. Special Issue for ANFC 2014, 2015. http://dx.doi.org/10.1080/00223131.2015.1021285

[18] D. Hasselman, L. Johnson, "Effective thermal conductivity of composites with interfacial thermal barrier resistance,” J Comp. Mater. 21 (1987) 508.

[19] A. Every, Y. Tzou, D. Hasselman, R. Raj, "The effect of particle size on the thermal conductivity of ZnS/diamond composites,” Acta Metal. Mater. 40(1992) 123.

[20] Y. Benveniste, "Effective thermal conductivity of composites with a thermal contact resistance between the constituents: Nondilute case,” J Appl. Phys. 61 (1987) 2840. 
[21] K.L. Peddicord, M.E. Cunningham, A. Tripathi, "Porosity correction to thermal conductivity based on analytical temperature solutions,” Trans. Am. Nucl. Soc., 28 (1978) 548 .

[22] A. Williams, B. Leitch, N. Wang, "A microstructural model of the thermal conductivity of dispersion type fuels with a fuel matrix interaction layer," Nucl. Eng. Technol. 45(2013) 839-846. 
Table 1 Thermal conductivity data for U-Mo/Al dispersion used in this study

\begin{tabular}{|c|c|c|c|}
\hline & $\begin{array}{l}\text { U-Mo alloy } \\
\text { composition }^{\text {a }}\end{array}$ & $\begin{array}{c}\text { Nominal U-Mo } \\
\text { volume fraction } \\
\text { in sample } \\
(\%)\end{array}$ & $\begin{array}{l}\text { Thermal } \\
\text { conductivity } \\
(\mathrm{W} / \mathrm{m}-\mathrm{K})\end{array}$ \\
\hline \multirow{12}{*}{$\begin{array}{l}\text { KAERI } \\
\text { data }^{b}\end{array}$} & \multirow{4}{*}{ U-6Mo } & 10 & $183.1-200.8$ \\
\hline & & 30 & $131.3-150.4$ \\
\hline & & 40 & $107.5-128.6$ \\
\hline & & 50 & $82.5-100.8$ \\
\hline & \multirow{4}{*}{ U-8Mo } & 10 & $180.3-195.9$ \\
\hline & & 30 & $124.2-139.3$ \\
\hline & & 40 & $93.9-112.3$ \\
\hline & & 50 & $66.8-85.3$ \\
\hline & \multirow{4}{*}{ U-10Mo } & 10 & $197.2-241.1$ \\
\hline & & 30 & $128.7-146.7$ \\
\hline & & 40 & $96.5-115.5$ \\
\hline & & 50 & $73.0-94.6$ \\
\hline \multirow{2}{*}{$\begin{array}{l}\text { ANL } \\
\text { data }^{c}\end{array}$} & \multirow{2}{*}{ U-10Mo } & 39 & $65.8-96.7$ \\
\hline & & 50 & $89.0-129.8$ \\
\hline
\end{tabular}

a The number in front of Mo stands for the Mo composition in the alloy in weight percent.

b The temperature range for the KAERI data is $25-380{ }^{\circ} \mathrm{C}$ [8]. The KAERI data was based on samples fabricated with U-Mo atomized powders.

c The temperature range for the ANL data is $23-500{ }^{\circ} \mathrm{C}$ [9]. The ANL data was measured for samples fabricated with U-Mo ground powders. 
Table 2 Recommended values of Al linear thermal expansion [15]

\begin{tabular}{c|c}
\hline $\begin{array}{c}\text { Temperature } \\
(\mathrm{K})\end{array}$ & $\begin{array}{c}\text { Linear thermal } \\
\text { expansion, } \Delta \mathrm{L}^{\mathrm{L}} \mathrm{L}_{0}(\%)\end{array}$ \\
\hline 293 & 0.000 \\
\hline 400 & 0.259 \\
\hline 500 & 0.514 \\
\hline 600 & 0.787 \\
\hline 700 & 1.084 \\
\hline 800 & 1.408 \\
\hline 900 & 1.764 \\
\hline
\end{tabular}


Table 3 Fitting constants for the interfacial thermal resistance in Eq. (16)

\begin{tabular}{c|c|c}
\hline & $\mathrm{a}\left(\mathrm{m}^{2}-\mathrm{K} / \mathrm{W}\right)$ & $\mathrm{b}(\mathrm{K})$ \\
\hline $\begin{array}{c}\text { For the interfacial thermal } \\
\text { resistance between U-Mo and Al }\end{array}$ & 0.01 & 0.01 \\
\hline $\begin{array}{c}\text { For the interfacial thermal } \\
\text { resistance between Al grains }\end{array}$ & 0.005 & 0.0002 \\
\hline
\end{tabular}




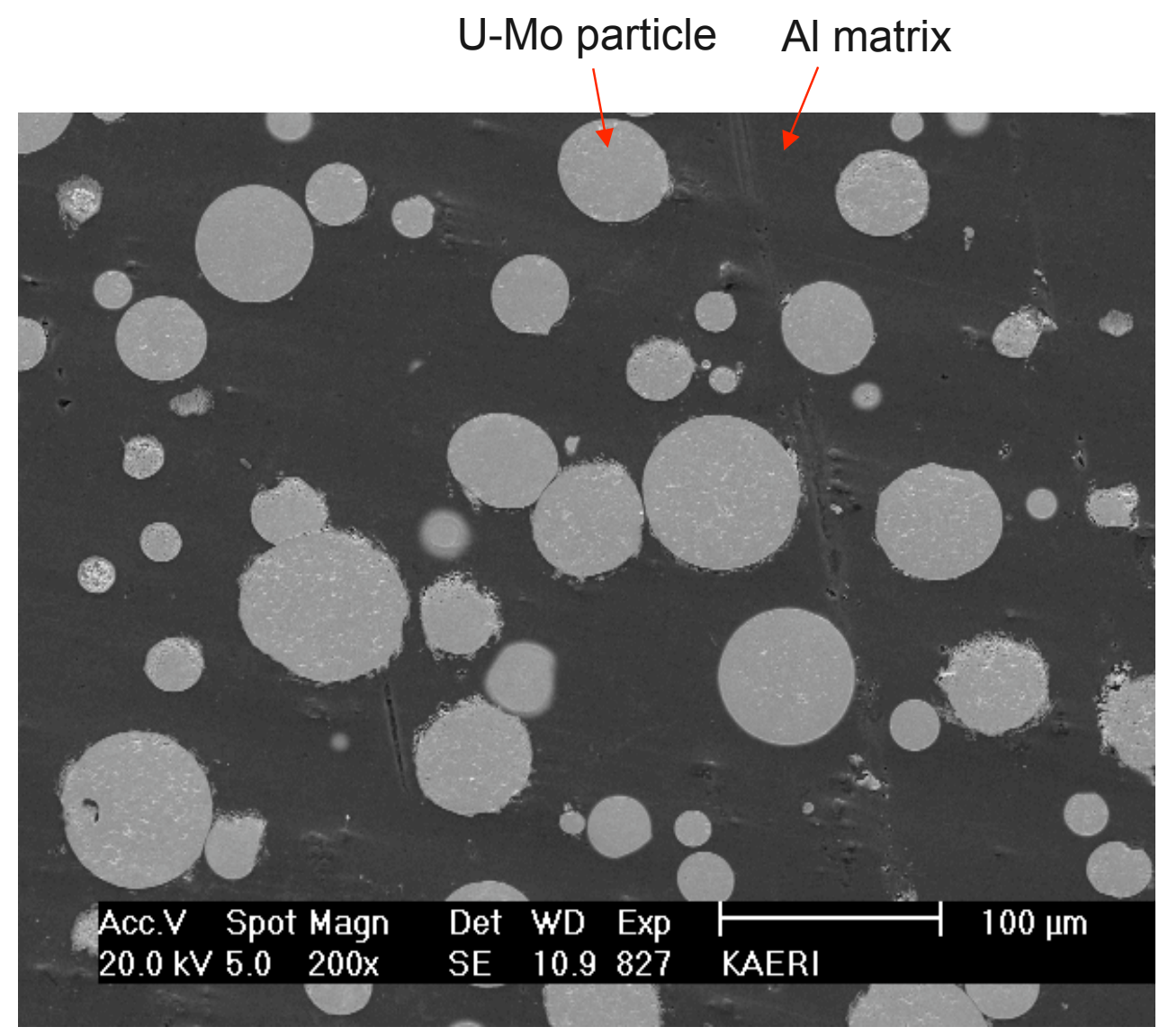

Fig. 1 SEM image showing a U-10Mo/Al dispersion fuel meat composed of U-Mo particles embedded in Al matrix. The U-10Mo volume fraction is $10 \%$ in the meat. This sample was fabricated by the co-extrusion method that was used to make the samples studied in the paper [3] and the samples to measure the KAERI data. 


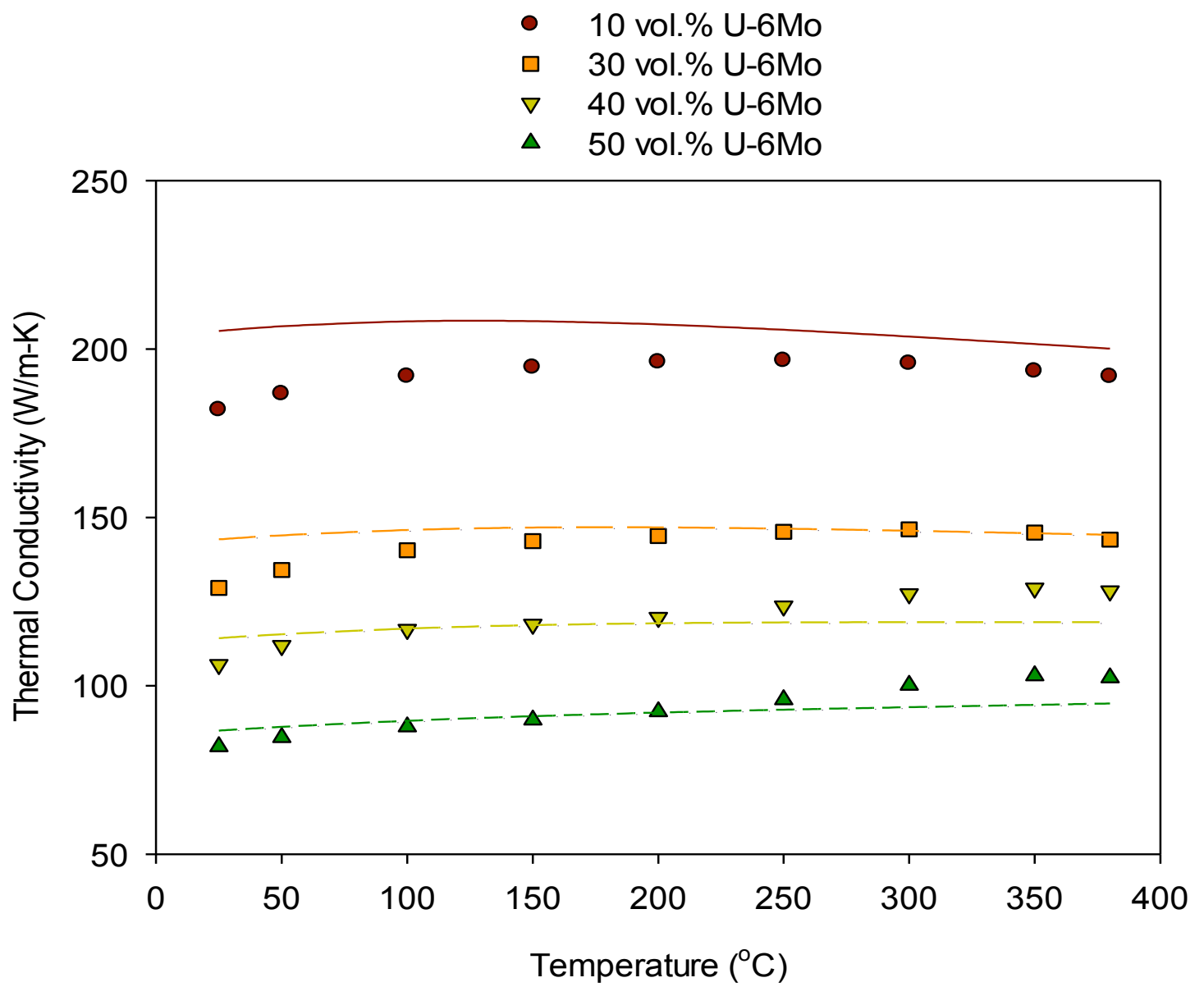

Fig. 2 Comparison between KAERI thermal conductivity data modified with temperature-dependent density and Bruggeman model predictions for $\mathrm{U}-6 \mathrm{Mo} / \mathrm{Al}$. The symbols are for the measured data and the lines are for the predictions. 


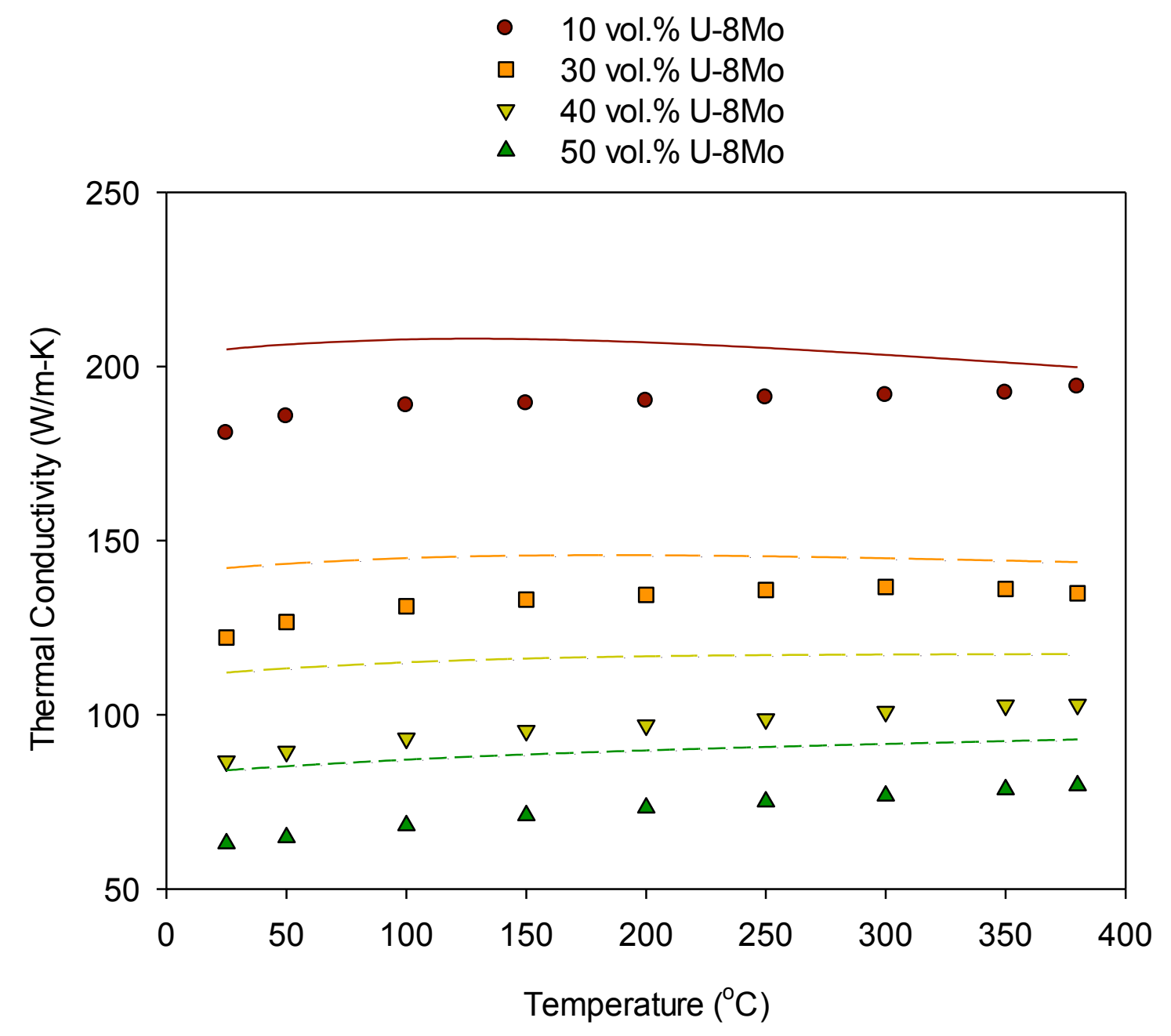

Fig. 3 Comparison between KAERI thermal conductivity data modified with temperature-dependent density and Bruggeman model predictions for $\mathrm{U}-8 \mathrm{Mo} / \mathrm{Al}$. The symbols are for the measured data and the lines are for the predictions. 


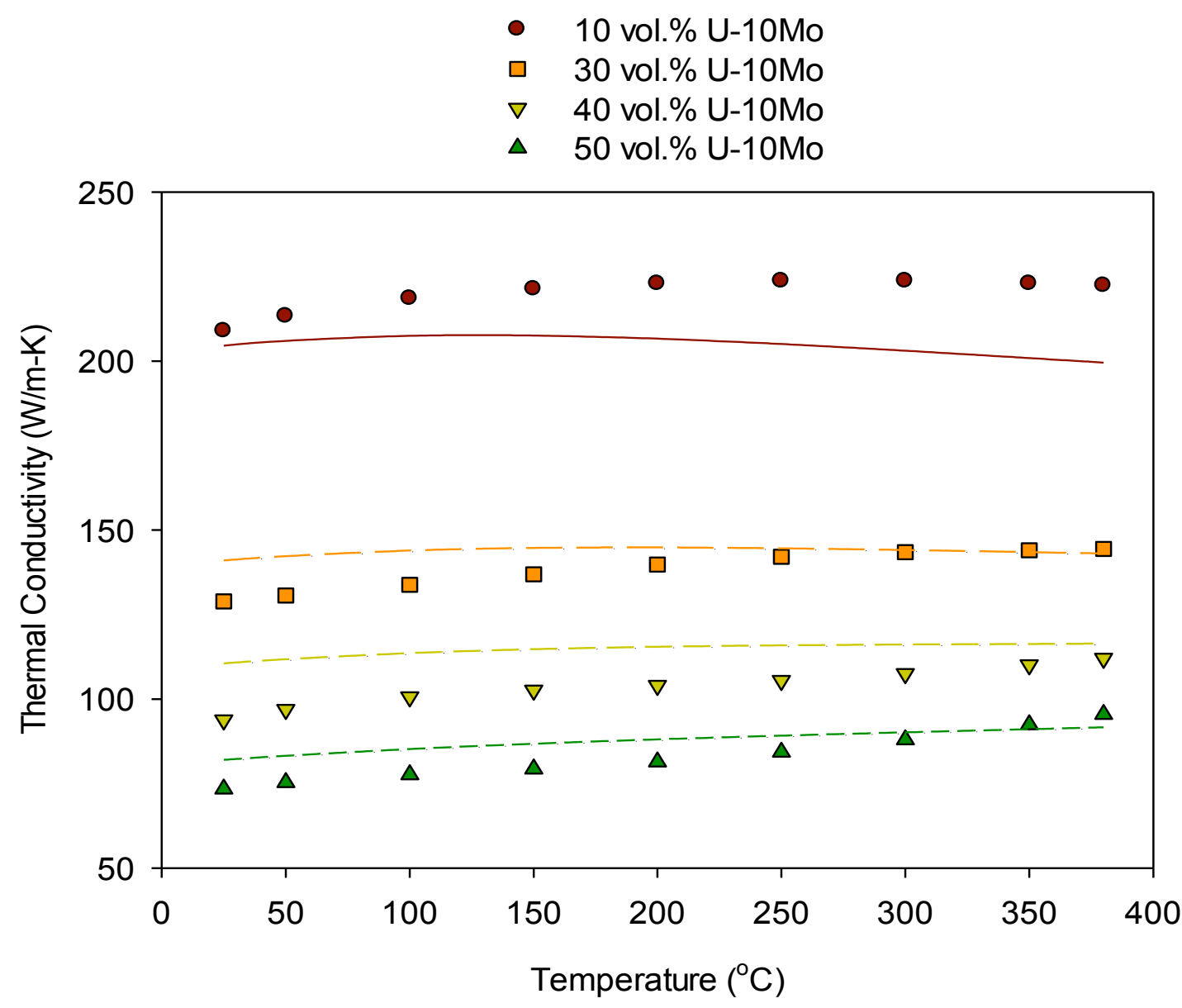

Fig. 4 Comparison between KAERI thermal conductivity data modified with temperature-dependent density and Bruggeman model predictions for U-10Mo/Al. The symbols are for the measured data and the lines are for the predictions. 


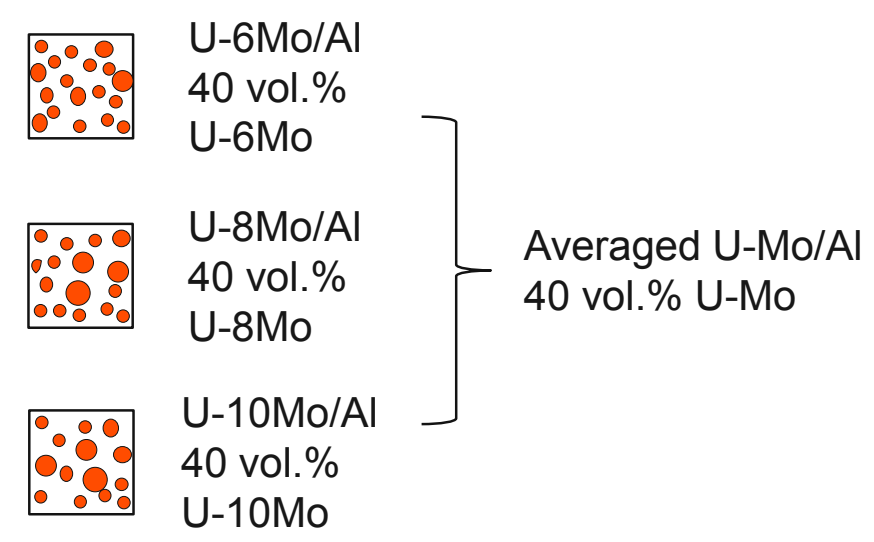

Fig. 5 Schematic illustrating the averaging method U-Mo samples with different Mo content in the U-Mo particles. The 40 vol.\% case is shown here for example. 


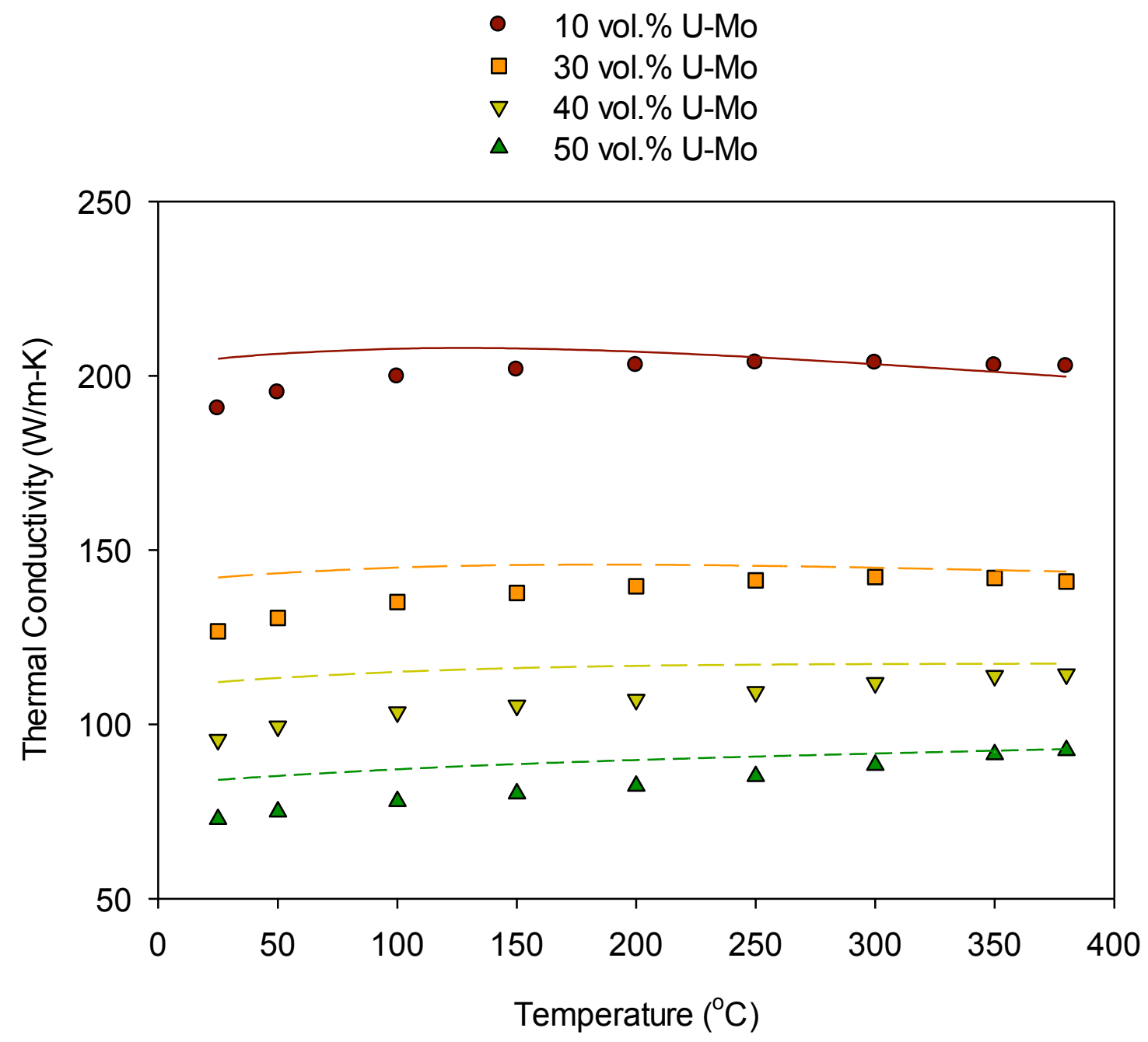

Fig. 6 KAERI thermal conductivity data modified to incorporate temperaturedependent density and the averaging method for thermal diffusivity. The lines are for the predictions using the Bruggeman model for $\mathrm{U}-8 \mathrm{Mo} / \mathrm{Al}$. 


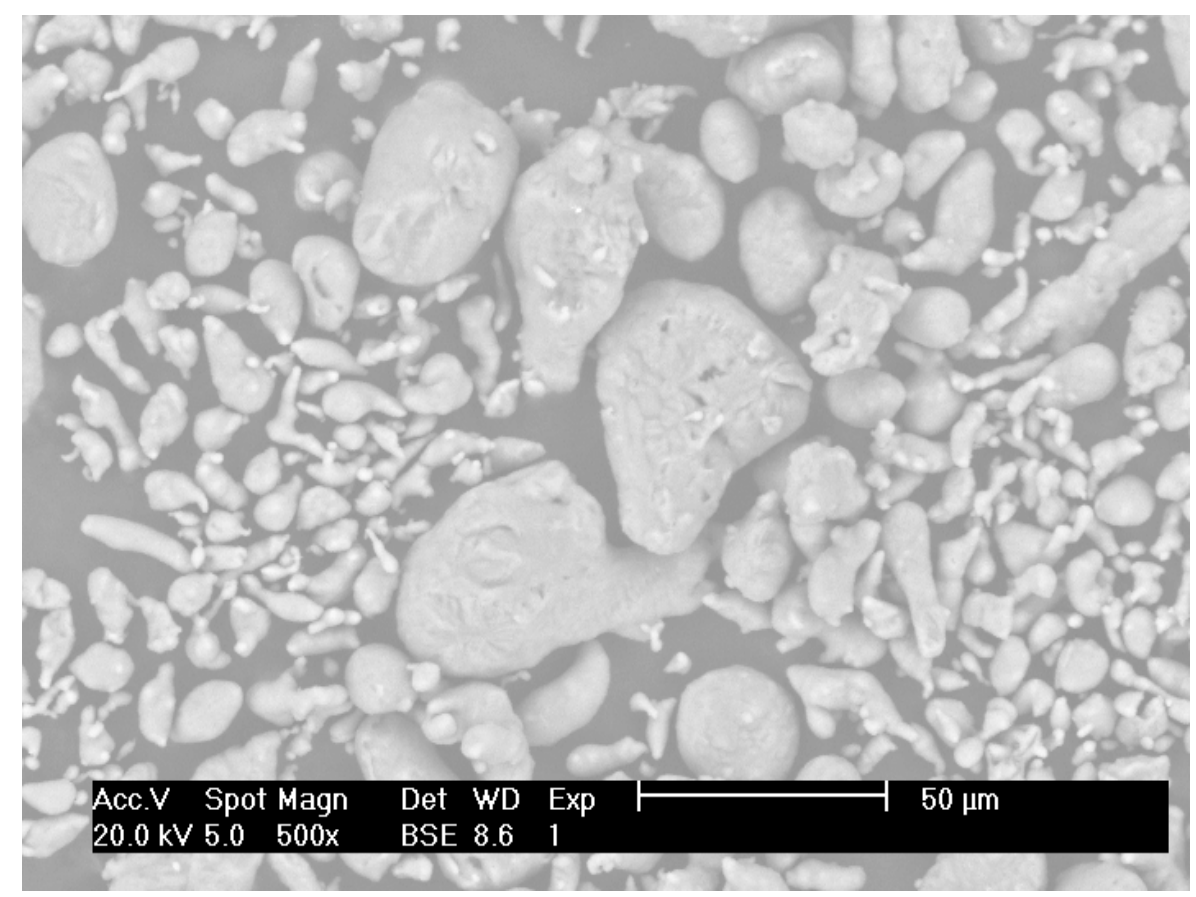

Fig. 7 SEM image of an Al powder used for the matrix for U-Mo/Al samples 
\begin{tabular}{ll}
- & 10 vol. $\%$ U-Mo \\
\hline & 30 vol. $\%$ U-Mo \\
$\nabla$ & 40 vol. $\%$ U-Mo \\
$\Delta$ & 50 vol. $\%$ U-Mo
\end{tabular}

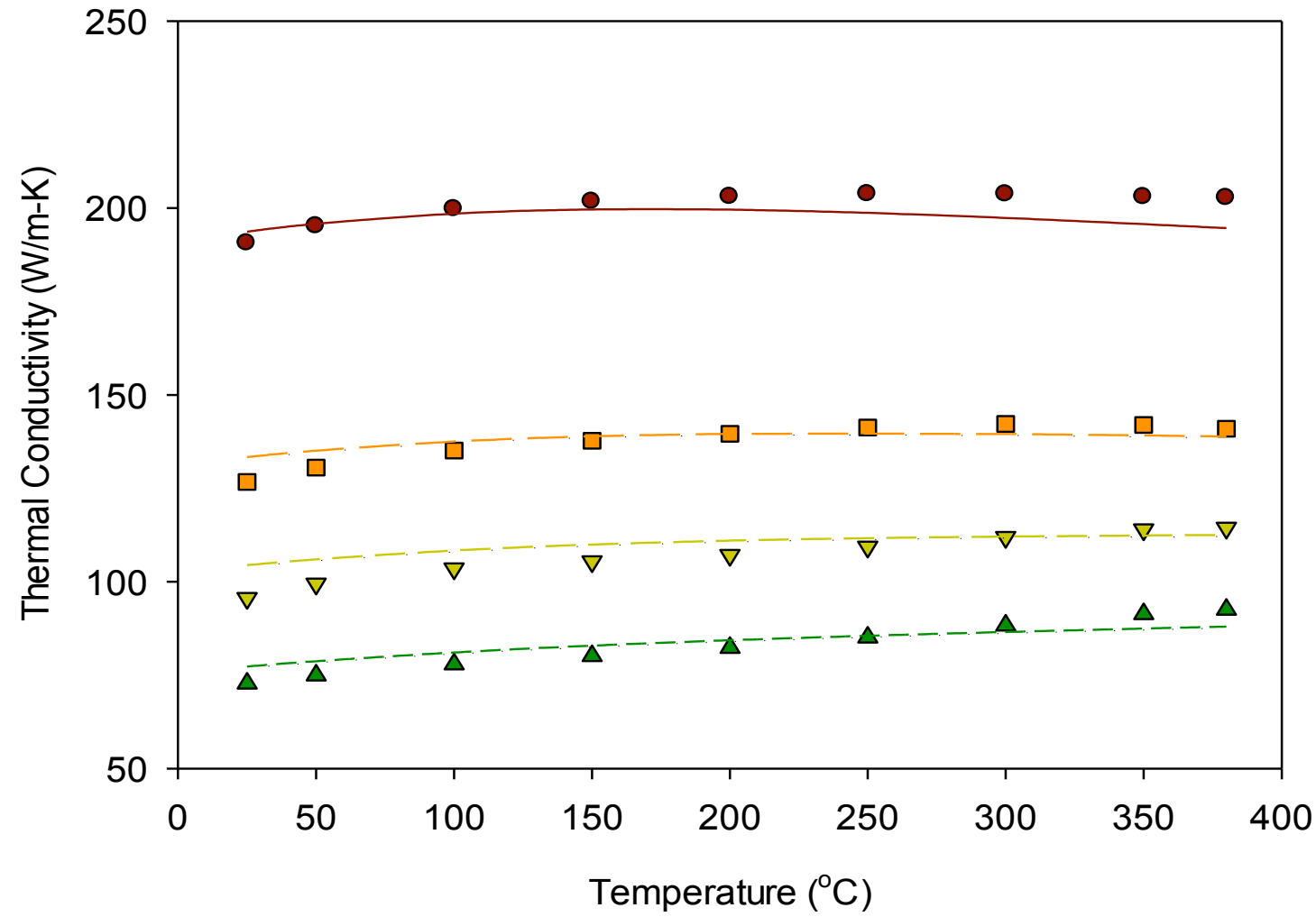

Fig. 8 Modified Bruggeman model predictions (solid lines) compared with KAERI thermal conductivity data modified with temperature-dependent density and averaging the data for $\mathrm{U}-6 \mathrm{Mo} / \mathrm{Al}, \mathrm{U}-8 \mathrm{Mo} / \mathrm{Al}$ and $\mathrm{U}-10 \mathrm{Mo} / \mathrm{Al}$ samples for the same U-Mo volume fraction (symbols). 


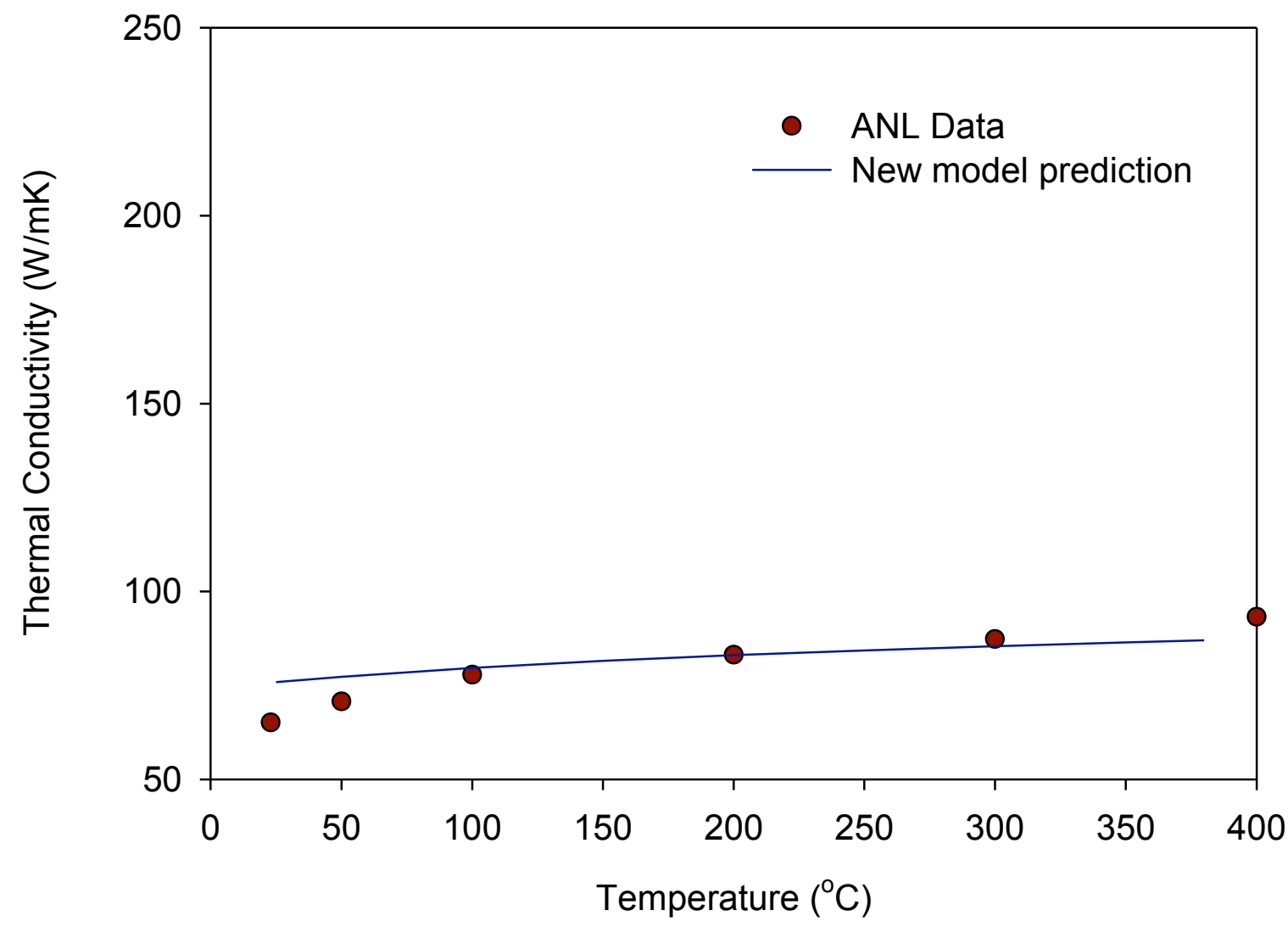

Fig. 9 Comparison of the new model prediction with the ANL data for 50 vol.\% U-Mo. 